Mappemonde

Revue trimestrielle sur l'image géographique et les formes du territoire

$120 \mid 2017$

Varia

\title{
Le palmarès du concours des posters scientifiques (Festival international de géographie)
}

\section{Antoine Fleury}

\section{OpenEdition}

\section{Journals}

Édition électronique

URL : https://journals.openedition.org/mappemonde/3114

DOI : 10.4000/mappemonde.3114

ISSN : 1769-7298

Éditeur

UMR ESPACE

\section{Référence électronique}

Antoine Fleury, « Le palmarès du concours des posters scientifiques (Festival international de géographie) », Mappemonde [En ligne], 120 | 2017, mis en ligne le 01 avril 2017, consulté le 28 juin 2022. URL : http://journals.openedition.org/mappemonde/3114 ; DOI : https://doi.org/10.4000/ mappemonde.3114

Ce document a été généré automatiquement le 29 septembre 2020.

\section{cc) (†)}

La revue Mappemonde est mise à disposition selon les termes de la Licence Creative Commons Attribution - Pas d'Utilisation Commerciale - Partage dans les Mêmes Conditions 4.0 International. 


\title{
Le palmarès du concours des posters scientifiques (Festival international de géographie)
}

\author{
Antoine Fleury
}

$1 \quad$ La $27^{\mathrm{e}}$ édition du Festival international de géographie (FIG) de Saint-Dié-des-Vosges s'est tenue du 30 septembre au 2 octobre 2016 avec pour thème « Un Monde qui va plus vite ? » et pour pays invité, la Belgique. Dans le cadre des « Vitrines de la géographie » du FIG, les expositions scientifiques présentent les travaux de géographes sous la forme de posters portant soit sur le thème, soit sur le pays invité. Elles constituent une vitrine reconnue des recherches actuelles en géographie tout en contribuant à la reconnaissance du poster comme support à part entière de la communication scientifique. En 2016, les expositions scientifiques ont rassemblé 19 posters proposés par des doctorants, des chercheurs et des enseignants-chercheurs venus de France, de Belgique, du Brésil ou encore de Turquie, dont quatre portaient sur le pays invité. Six posters produits dans le cadre de projets pédagogiques par des élèves du secondaire ont également été exposés, ce qui a constitué une innovation du FIG2016.

Doté par l'ADFIG (Association pour le Développement du FIG), le prix du meilleur poster scientifique est décerné par un jury dont les membres ont des spécialités complémentaires. En 2016, ce jury était composé de Béatrice Collignon (université Bordeaux Montaigne), Martine Drozdz (CNRS), Antoine Fleury (CNRS) et Cyrille Suss (cartographe indépendant). Trois posters ont été récompensés pour leur qualité de réalisation, tant sur le fond que sur la forme. Ils ont en particulier retenu l'attention du jury pour la qualité des documents iconographiques et du travail de composition, pour la clarté et la lisibilité du message scientifique, ainsi que pour la pertinence et l'originalité de la recherche présentée.

3 Comme chaque année, M@ppemonde publie les travaux primés, accompagnés d'un texte de présentation des auteur-es :

- $1^{\text {er }}$ prix : «Être éleveur, dans un monde qui va toujours plus vite : course contre le temps ou temps contre-nature ?* » par Marie Guicheteau (CERAMAC, Université Clermont Auvergne). 
- $2^{\mathrm{e}}$ prix : «L'altitude ralentit le déclin? 40 ans de présence des épiceries dans les communes rurales et les bourgs isolés » par Sophie Baudet-Michel, Ludovic Chalonge et Constance Lecomte (Géographie-cités, CNRS/Université Paris 1 Panthéon-Sorbonne/Université Paris Diderot)

- $3^{\mathrm{e}}$ prix : «Le maillage communal en Belgique : des héritages différenciés » par Jean-Baptiste Grison (CERAMAC, Université Clermont Auvergne)

Depuis sa création, l'un des objectifs du concours est de réfléchir et de travailler avec les auteur-es à la meilleure manière de construire des posters scientifiques. C'est pourquoi le jury a transmis aux auteur-es des posters primés un certain nombre de suggestions destinées à améliorer encore leur travail. Les posters publiés ici ont donc été légèrement remaniés par rapport à la version exposée à Saint-Dié-des-Vosges.

*Ce poster sera publié ultérieurement.

INDEX

Keywords : FIG, FIG2016

\section{AUTEUR}

\section{ANTOINE FLEURY}

Chargé de Recherche au CNRS 